\title{
Applying fractional calculus to analyze final consumption and gross investment influence on GDP
}

\author{
A. BADÍK AND M. FEČKAN
}

\begin{abstract}
This paper points out the possibility of suitable use of Caputo fractional derivative in regression model. Fitting historical data using a regression model seems to be useful in many fields, among other things, for the short-term prediction of further developments in the state variable. Therefore, it is important to fit the historical data as accurately as possible using the given variables. Using Caputo fractional derivative, this accuracy can be increased in the model described in this paper.
\end{abstract}

Mathematics Subject Classification 2010: 26A33, 26A51, $26 \mathrm{D} 15$.

Keywords: Caputo derivative, Method of least squares, Regression model.

\section{INTRODUCTION}

Fractional (fractional-order) derivative also known as derivative with memory appears to be a powerful tool for examining the development of economic indicators (such as GDP) because of 'memory'. It has been proved that fractional models [Hilfer et al. 2000] are better then integer models, giving us a great opportunity to use it. It turns out that fractional calculus is also advantageous to use in regression models, which then provide better accuracy for modeling variable of interest based on a set of predictor variables than can be seen in [Luo et al. 2018].

In [Anghelache et al. 2015], the authors study GDP evolution for the Romanian case by using multiple linear regression model with final consumption value and the value of gross investment as independent variables via data between 1990 and 2014.

In the present paper, we go on the study of final consumption and gross investment influence on GDP for the Romanian case by using Caputo fractional derivative. We note that the Romanian case is used in this paper only for possibility to compare our achievement and proposed model with previous one.

Partially supported by the Slovak Research and Development Agency under the contract No. APVV-180308 and by the Slovak Grant Agency VEGA No. 1/0358/20 and No. 2/0127/20. 


\section{APPROXIMATION TO CAPUTO FRACTIONAL DERIVATIVE BASED ON POLYNOMIAL INTERPOLATION}

From the definition of the Caputo derivative, we can find that the $\alpha$ th-order ( $m-1<\alpha<m$ ) Caputo derivative of the given function $f(t)$ can be seen as the $(m-\alpha)$ th-order fractional integral of the function $f^{(m)}(t)$. In our case, we decided to use fractional rectangular formula.

For $0<\alpha<1$ we get the following formula

$$
\left[{ }_{C} D_{0, t}^{\alpha} f(t)\right]_{t=t_{n}} \approx \sum_{k=0}^{n-1} w_{n-k-1} \delta_{t} f\left(t_{k}\right)
$$

where

$$
w_{i}=\frac{\Delta t^{1-\alpha}}{\Gamma(2-\alpha)}\left[(i+1)^{1-\alpha}-i^{1-\alpha}\right]
$$

and

$$
\delta_{t} f\left(t_{k}\right)=\frac{f\left(t_{k+1}\right)-f\left(t_{k}\right)}{\Delta t} \approx f^{\prime}\left(t_{k}\right) .
$$

\section{REGRESSION MODELS WITH FRACTIONAL-ORDER DERIVATIVES}

Throughout of this paper, we denote GDP by $y$, final consumption by $x_{1}$ and gross investments by $x_{2}$.

The modification of original model leads to

$$
y(t)=\beta_{0}+\beta_{1} D_{t_{0}, t}^{\alpha_{1}} x_{1}(t)+\beta_{2} C_{t_{0}, t}^{\alpha_{2}} x_{2}(t)+\varepsilon(t),
$$

where $\beta_{i}(i=0,1,2)$ are regression coefficients, $\alpha_{1}, \alpha_{2} \in[0,1]$ are unknown orders and $\varepsilon(t)$ is a function of residuals.

We also denote the mean square error by MSE, the coefficient of determination by $R^{2}$, the adjusted coefficient of determination by $\bar{R}^{2}$, the mean absolute deviation by MAD and Akaike Information Criterion by AIC.

Define

$$
\begin{aligned}
& \mathrm{MSE}=\frac{1}{n} \sum_{i=0}^{n}\left(y_{i}-\hat{y}_{i}\right)^{2}, \\
& R^{2}=1-\frac{\sum_{i=0}^{n}\left(y_{i}-\hat{y}_{i}\right)^{2}}{\sum_{i=0}^{n}\left(y_{i}-\bar{y}\right)^{2}},
\end{aligned}
$$




$$
\begin{gathered}
\bar{R}^{2}=1-\left(1-R^{2}\right) \frac{n-1}{n-k-1}, \\
\operatorname{MAD}=\frac{1}{n} \sum_{i=0}^{n}\left|y_{i}-\hat{y}_{i}\right|, \\
\mathrm{AIC}=n \ln \left(\frac{1}{n} \sum_{i=0}^{n}\left(y_{i}-\hat{y}_{i}\right)^{2}\right)+2 k+\frac{2 k(k+1)}{n-k-1},
\end{gathered}
$$

where $n$ denotes the sample size and $k$ denotes the number of parameters.

\subsection{Results}

The least squares method gives the following estimates of coefficients and orders of the fractional operators (see Table I).

\begin{tabular}{ccc}
\hline & original model & model (2) \\
\hline$\beta_{0}$ & -2.14384 & 82.43610 \\
$\beta_{1}$ & 1.16311 & 1.34269 \\
$\beta_{2}$ & 0.32493 & -10.88530 \\
\hline \hline$\alpha_{1}$ & - & $4.41093 \times 10^{-17}$ \\
$\alpha_{2}$ & - & 0.58188 \\
\hline
\end{tabular}

Table I. Estimates of coefficients and orders of the fractional operators.

Results obtained by using (1)-(2) are as follows (see Table II).

\begin{tabular}{lcc}
\hline & original model & model (2) \\
\hline MSE & 22.8812 & 19.9679 \\
$R^{2}$ & 0.98308 & 0.98523 \\
$\bar{R}^{2}$ & 0.98154 & 0.98389 \\
MAD & 4.21674 & 3.82673 \\
AIC & 85.3488 & 81.9441 \\
\hline
\end{tabular}

Table II. Performance indices for the Romanian economy.

Now, when we know the coefficients and orders of the fractional operators, we are ready to give the fitting results (see Figure 1). 


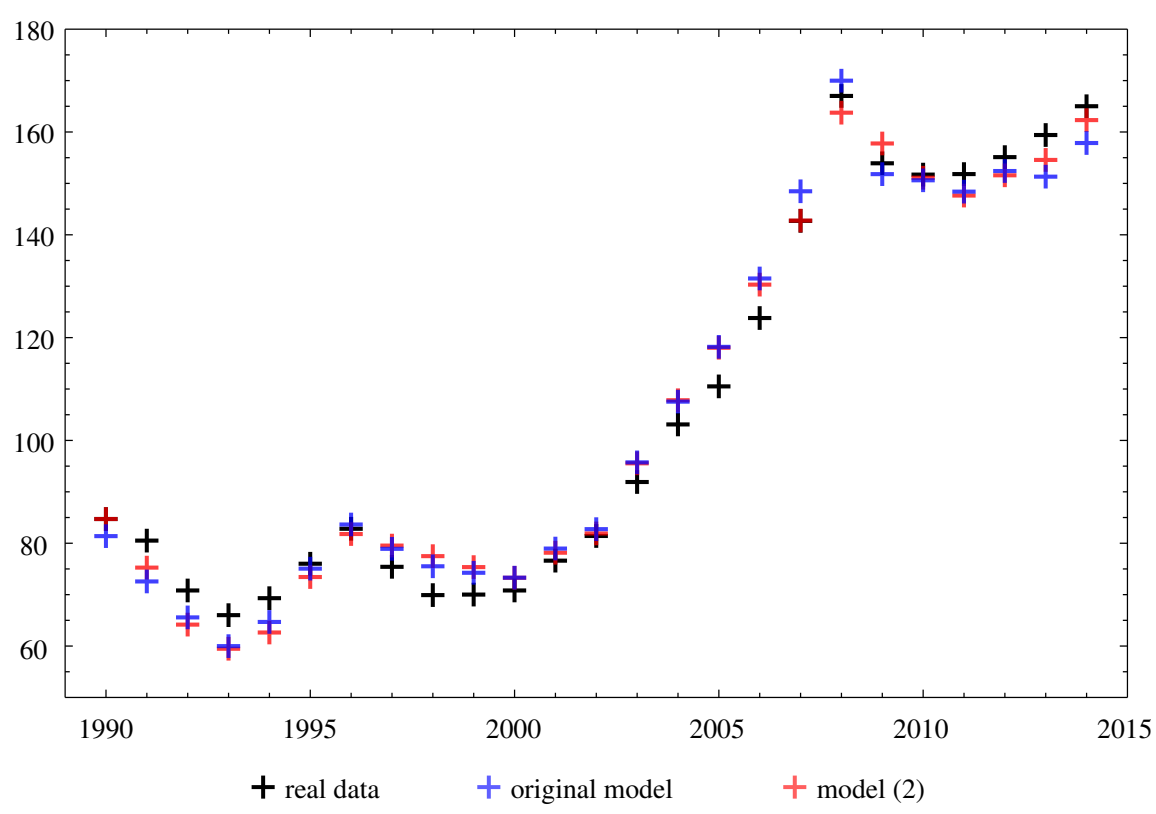

Fig. 1. Data fitting.

One can see that the simulation results of original and model (2) are close to real data. However, $R^{2}$ of modified model is closer to 1 than $R^{2}$ of original model. Thus, model (2) is better than original model.

\subsection{More General Models}

We consider extension of (2) in a form

$$
\begin{aligned}
y(t)=\beta_{0} & +\beta_{1,1 C} D_{t_{0}, t}^{\alpha_{1,1}} x_{1}(t)+\beta_{1,2} D_{t_{0}, t}^{\alpha_{1,2}} x_{1}(t) \\
& +\beta_{2,1 C} D_{t_{0}, t}^{\alpha_{2,1}} x_{2}(t)+\beta_{2,2} D_{t_{0}, t}^{\alpha_{2,2}} x_{2}(t)+\varepsilon(t),
\end{aligned}
$$

where $\beta_{0}, \beta_{1, i}, \beta_{2, i}(i=0,1,2)$ are regression coefficients, $\alpha_{1, i}, \alpha_{2, i} \in[0,1](i=0,1,2)$ are unknown orders and $\varepsilon(t)$ is a function of residuals. In general, we have

$$
y(t)=\beta_{0}+\sum_{i=1}^{p} \beta_{1, i C} D_{t_{0}, t}^{\alpha_{1, i}} x_{1}(t)+\sum_{j=1}^{q} \beta_{2, j C} D_{t_{0}, t}^{\alpha_{2, j}} x_{2}(t)+\varepsilon(t)
$$

for $\alpha_{1, i}, \alpha_{2, j} \in[0,1], i=1, \ldots, p, j=1, \ldots, q$. Letting $p \rightarrow \infty$ and $q \rightarrow \infty$, we get

$$
y(t)=\beta_{0}+\int_{0}^{1} \beta_{1}\left(\alpha_{1}\right)_{C} D_{t_{0}, t}^{\alpha_{1}} x_{1}(t) d \alpha_{1}+\int_{0}^{1} \beta_{2}\left(\alpha_{2}\right)_{C} D_{t_{0}, t}^{\alpha_{2}} x_{2}(t) d \alpha_{2}+\varepsilon(t) .
$$


Due to the computational complexity of the least squares method for all parameters, we chose a different method for estimating the models parameters and we get the following estimates of coefficients and orders of the fractional operators (see Table III). Note that there is no proof of the best estimate of parameters obtained using this method.

\begin{tabular}{lcccc}
\hline & model (3) & model $(4), p=q=3$ & model $(4), p=q=4$ & model $(4), p=q=5$ \\
\hline$\beta_{0}$ & 83.20526 & 84.04196 & 85.22125 & 85.15662 \\
$\beta_{1,1}$ & 1.47409 & 19.39253 & 72.97881 & 31.97668 \\
$\beta_{1,2}$ & -1.27170 & -40.21083 & -169.16983 & -60.53592 \\
$\beta_{1,3}$ & - & 52.15342 & 303.43792 & -9.41558 \\
$\beta_{1,4}$ & - & - & -2333.38776 & -33494.91513 \\
$\beta_{1,5}$ & - & - & - & 25613.50872 \\
$\beta_{2,1}$ & -4.15815 & 421.22459 & 2453.17281 & -124.40979 \\
$\beta_{2,2}$ & 3.28790 & -103.72563 & -556.75607 & -30.71726 \\
$\beta_{2,3}$ & - & -341.06431 & -2106.16932 & 264.10137 \\
$\beta_{2,4}$ & - & - & 839.70531 & -2685.75423 \\
$\beta_{2,5}$ & - & - & - & 9747.93546 \\
\hline \hline$\alpha_{1,1}$ & $3.1716 \times 10^{-11}$ & $1.4432 \times 10^{-14}$ & $8.2771 \times 10^{-10}$ & $1.49024 \times 10^{-9}$ \\
$\alpha_{1,2}$ & 0.09639 & 0.08246 & 0.08246 & 0.08246 \\
$\alpha_{1,3}$ & - & 0.35410 & 0.35408 & 0.35413 \\
$\alpha_{1,4}$ & - & - & 0.99967 & 0.99967 \\
$\alpha_{1,5}$ & - & - & - & 0.94407 \\
$\alpha_{2,1}$ & 0.07450 & 0.07450 & 0.07450 & 0.07450 \\
$\alpha_{2,2}$ & $1.0001 \times 10^{-20}$ & 0.00561 & 0.00561 & 0.00561 \\
$\alpha_{2,3}$ & - & 0.11702 & 0.11702 & 0.11702 \\
$\alpha_{2,4}$ & - & - & 0.68561 & 0.68561 \\
$\alpha_{2,5}$ & - & - & - & 0.99984 \\
\hline & & & &
\end{tabular}

Table III. Estimates of coefficients and orders of the fractional operators. 
Results obtained by using (1)-(4) are as follows (see Table IV).

\begin{tabular}{lccc}
\hline & original model & model $(2)$ & model (3) \\
\hline MSE & 22.8812 & 19.9679 & 6.8990 \\
$R^{2}$ & 0.98308 & 0.98523 & 0.99490 \\
$\bar{R}^{2}$ & 0.98154 & 0.98390 & 0.99388 \\
MAD & 4.21674 & 3.82673 & 2.12068 \\
AIC & 85.3488 & 81.9441 & 58.2843 \\
\hline & & & \\
\hline & model $(4), p=q=3$ & model $(4), p=q=4$ & model $(4), p=q=5$ \\
\hline MSE & 3.53354 & 1.51559 & 1.45406 \\
$R^{2}$ & 0.99739 & 0.99888 & 0.99893 \\
$\bar{R}^{2}$ & 0.99652 & 0.99832 & 0.99816 \\
MAD & 1.42666 & 0.96946 & 0.93319 \\
AIC & 48.2242 & 35.3951 & 45.0733 \\
\hline
\end{tabular}

Table IV. Performance indices for the Romanian economy.

Now, when we know the coefficients and orders of the fractional operators, we are ready to give the fitting results (see Figure 2).
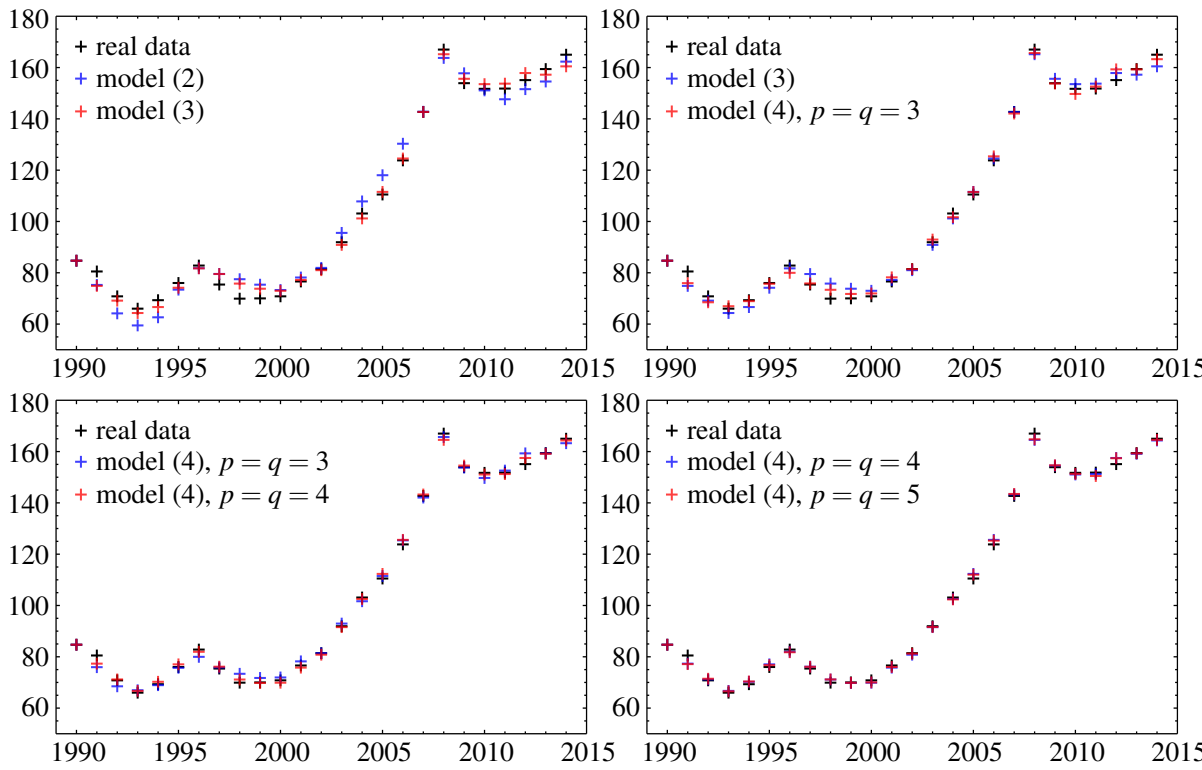

Fig. 2. Data fitting. 
The simulation results of models where we estimated the parameters are close to real data. Due to the different count of parameters of models, we can use adjusted coefficient of determination for the model comparison to each other.

One can see, that data obtained by model (4) with $p=q=4$ better fits real data than data obtained by other models, which is also indicated by values of $\bar{R}^{2}$ of these models. $\bar{R}^{2}$ of model (4) with $p=q=4$ is closer to 1 than $\bar{R}^{2}$ of any other model where we estimated the parameters. Thus, model (4) with $p=q=4$ is better then any other model where we estimated the parameters.

\section{CONCLUSIONS}

This paper studies a final consumption and gross investment influence on GDP for the Romanian case. Based on our results, it is shown that using the Caputo fractional derivative is convenient in this case. In addition, the data of general models are better than the data of original model from [Anghelache et al. 2015].

\section{REFERENCES}

Anghelache, C., Manole, A., Anghel, M. G., ET Al. 2015. Analysis of final consumption and gross investment influence on GDP - multiple linear regression model. Theoretical and Applied Economics 22, $137-142$.

DiETHELM, K. 2010. The analysis of fractional differential equations: An application-oriented exposition using differential operators of Caputo type. Springer Science \& Business Media.

HILFER, R. ET AL. 2000. Applications of fractional calculus in physics. World scientific Singapore.

LI, C. AND ZENG, F. 2015. Numerical methods for fractional calculus. Chapman and Hall/CRC.

LUO, D., WANG, J., AND FEČKAN, M. 2018. Applying fractional calculus to analyze economic growth modelling. Journal of Applied Mathematics, Statistics and Informatics 14, 1, 25-36.

Ortigueira, M. D. AND Machado, J. T. 2015. What is a fractional derivative? Journal of computational Physics 293, 4-13.

PodlubNy, I. 1998. Fractional differential equations: an introduction to fractional derivatives, fractional differential equations, to methods of their solution and some of their applications. Elsevier.

ZHOU, Y. 2016. Fractional evolution equations and inclusions: Analysis and control. Academic Press. 
Andrej Badík

Department of Mathematical Analysis and Numerical Mathematics

Faculty of Mathematics, Physics and Informatics

Comenius University in Bratislava

email: andrej.badik@fmph.uniba.sk

Michal Fečkan

Department of Mathematical Analysis and Numerical Mathematics

Faculty of Mathematics, Physics and Informatics

Comenius University in Bratislava

email: michal.feckan@fmph.uniba.sk 